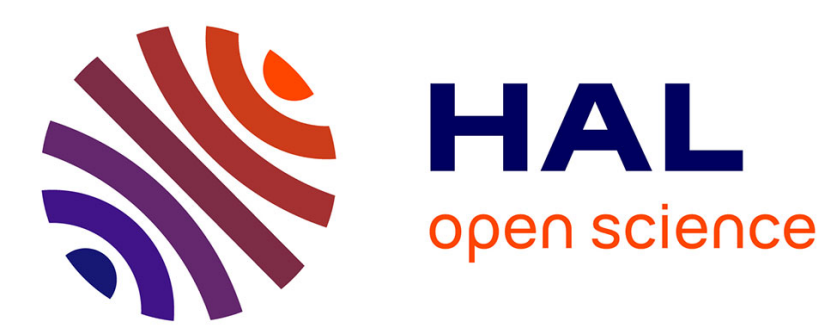

\title{
Control of the new 4th order hyper-chaotic system with one input
}

\author{
Antonio Loria
}

\section{To cite this version:}

Antonio Loria. Control of the new 4th order hyper-chaotic system with one input. Communications in Nonlinear Science and Numerical Simulation, 2010, 15 (6), pp.1621-1630. 10.1016/j.cnsns.2009.06.018 . hal-00521509

\section{HAL Id: hal-00521509}

https://hal-centralesupelec.archives-ouvertes.fr/hal-00521509

Submitted on 28 Mar 2011

HAL is a multi-disciplinary open access archive for the deposit and dissemination of scientific research documents, whether they are published or not. The documents may come from teaching and research institutions in France or abroad, or from public or private research centers.
L'archive ouverte pluridisciplinaire HAL, est destinée au dépôt et à la diffusion de documents scientifiques de niveau recherche, publiés ou non, émanant des établissements d'enseignement et de recherche français ou étrangers, des laboratoires publics ou privés. 


\title{
Control of the new $4^{\text {th }}$ order hyper-chaotic system with one input
}

\author{
Antonio Loría \\ CNRS, LSS-SUPELEC, 3, Rue Joliot Curie, 91192 Gif s/Yvette, France.
}

\begin{abstract}
We solve the problem of chaos suppression of Lü's hyper-chaotic system via feedback control. We use only one control input and moreover the controller is a simple proportional feedback and uses the measurement of only one variable. We show that this simple control law suffices to stabilize the hyper-chaotic system to the zero equilibrium globally and asymptotically. We present stability proofs based on Lyapunov's direct method and integration of solutions. As a corollary of our main result we draw the conclusion that the system is globally stabilizable by simply varying one parameter, when possible. Simulation experiments that show the effectiveness of our method are also presented.
\end{abstract}

PACS: 05.45.Ac; 05.45.Gg; 02.30.Hq; 05.45.Pq

Keywords: Lü system, Lorenz system, chaos control, Lyapunov method

\section{Introduction}

Many mathematical definitions of chaos exist but roughly, it may be described as a type of dynamic behaviour with the following characteristics: extreme sensitivity to changes in initial conditions, random-like behaviour, deterministic motion, trajectories of chaotic systems pass through any point an infinite number of times. The discovery of chaos as a dynamic behaviour goes back undoubtedly to the mathematician and physicist $\mathrm{H}$. Poincaré in the early 1900s, notably, in his celebrated work on the 3-body problem. Experimentally, chaos was first observed by Lorenz while simulating weather models. The celebrated Lorenz system [8] is a third-order autonomous system with two bilinear nonlinearities which produces the famous "butterfly attractor". In $[3,15]$, Chen generated a somewhat similar yet topologically different chaotic system, known now as the "Chen" system. One shall also mention the Rössler chaotic system - cf. [14] which, as the first two is bilinear and of 3rd order.

Another interesting 3rd-order chaotic system is the so-called Lü system [10]:

$$
\begin{aligned}
& \dot{x}=a(y-x) \\
& \dot{y}=-x z+c y \\
& \dot{z}=x y-b z
\end{aligned}
$$

Email address: loria@lss.supelec.fr (Antonio Loría). 
with $a=36, b=3$ and $c=20$. Parallel research on the Lü system has been carried on to produce other 3rd-order modifications of the latter with yet more interesting characteristics. For instance, the modified Lü system

$$
\begin{aligned}
& \dot{x}_{s}=\beta x_{s}-y_{s} z_{s}+c \\
& \dot{y}_{s}=-a y_{s}+x_{s} z_{s} \\
& \dot{z}_{s}=-b z_{s}+x_{s} y_{s}
\end{aligned}
$$

was introduced by the authors of [10] in [9]. Master-slave synchronization of this type of systems is studied via nonlinear feedback in $[13,16]$.

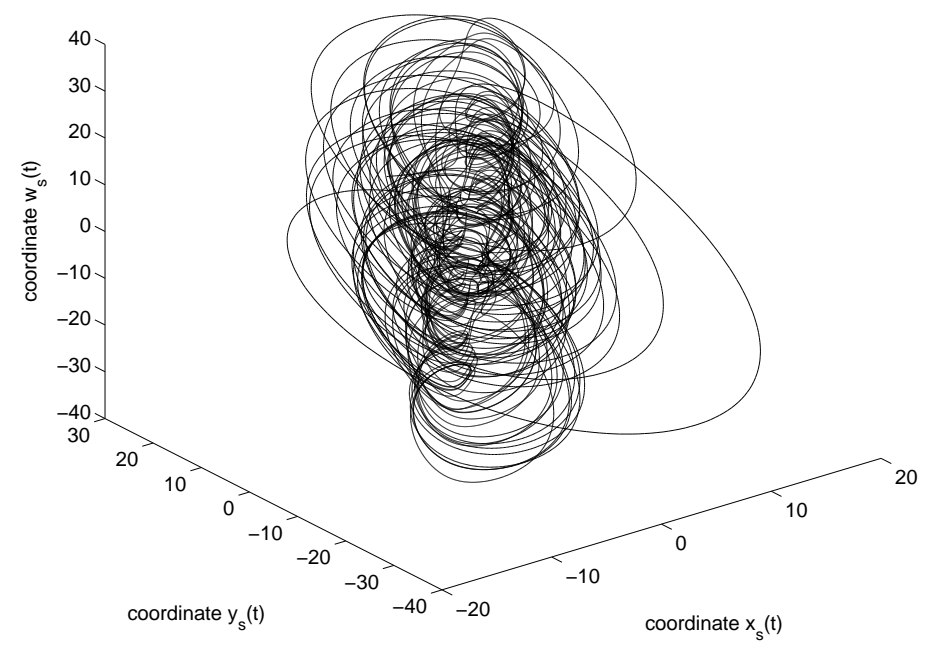

Fig. 1. Attractor of hyper-chaotic system without inputs i.e., $u_{x}=u_{y}=u_{w}=u_{z}=0$ - coordinates $x$ - $y$ - $z$

One of the aspects of undoubted interest in chaos research is control. For instance, the ability to make the system dynamics converge to a constant set-point, a periodic orbit or a different chaotic attractor than the natural one. Many techniques exist for chaos control such as the well-known OGY method [12], time-delay [6], nonlinear feedback control -cf. [11], sliding modes - $c f$. [2], Lyapunov exponents placement - $c f$. [1] to mention a few.

In this article we concentrate on chaos control of the Lü hyper-chaotic system. An interesting feature of this system is that by adding a feedback term and making the parameters vary appropriately one may provoke the system to exhibit different chaotic attractors; indeed, its behaviour will range from that similar to the Lorenz system to the Chen system. In the very recent article [7] the authors present an exhaustive study enriched with many simulation experiments that show how, via feedback control one may exhibit different chaotic regimes, going from those of the Lorenz system to the Chen's and other intermediary behaviors. The change of dynamics is provoked via feedback control, using different techniques: fuzzy control, nonlinear feedback control, etc. In particular, the latter consists on adding a bilinear nonlinearity to one of the system's equations and making a parameter vary over different ranges. 
Other articles where control of this hyper-chaotic system is studied include $[4,5,11]$. In [5] several control laws are proposed which stabilize the system at the zero equilibrium; for instance, a linear control law of dimension four and nonlinear feedbacks. In [11] the complex method of backstepping control is used to construct a highly nonlinear controller but which is also suitable to drive the system towards periodic orbits.

In this article we study the control towards an equilibrium point (hence chaos suppression) of the generalized 4th-order Lü system given by $-c f$. [5]

$$
\begin{aligned}
\dot{x}_{s} & =a\left(y_{s}-x_{s}\right)+u_{x} \\
\dot{y}_{s} & =b x_{s}-k x_{s} z_{s}+w_{s}+u_{y} \\
\dot{z}_{s} & =-c z_{s}+h x_{s}^{2}+u_{z} \\
\dot{w}_{s} & =-d x_{s}+u_{w}
\end{aligned}
$$

where $a, b, c, d, k$ and $h$ are constant parameters and $u_{x}, u_{y}, u_{z}$ and $u_{w}$ are control inputs. For the following values of these physical parameters, the unforced system that is, with all control inputs set to zero, exhibits a hyper-chaotic behaviour:

$$
a=10, b=40, c=2.5, d=10.6, k=1, h=4 .
$$

The attractors for the case when the system is unforced (i.e., with all controls set to zero) are showed in Figures 1,2 and 3. The initial conditions are set to:

$$
x_{s}(0)=-4 \quad y_{s}(0)=-8 \quad w_{s}(0)=12 \quad z_{s}(0)=-6 .
$$

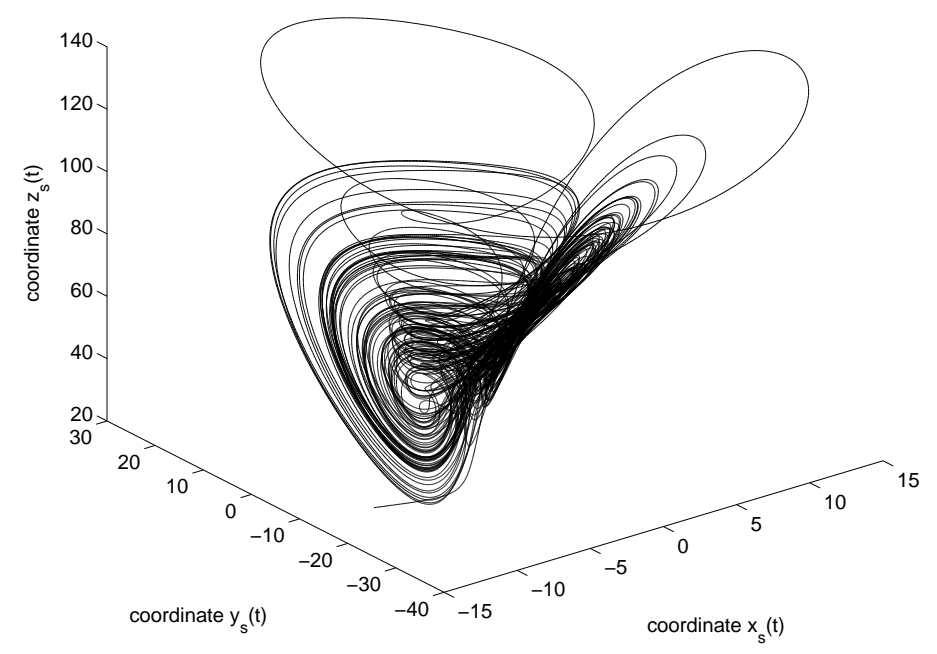

Fig. 2. Attractor of hyper-chaotic system without inputs $i . e ., u_{x}=u_{y}=u_{w}=u_{z}=0$ - coordinates $x-y$ - $w$

As mentioned, control to the origin of system (3) has been very recently studied in [5] where several techniques are proposed: the first uses linear proportional feedback on all four inputs; the second uses speed feedback of $\dot{y}$ which, to be implemented either requires 


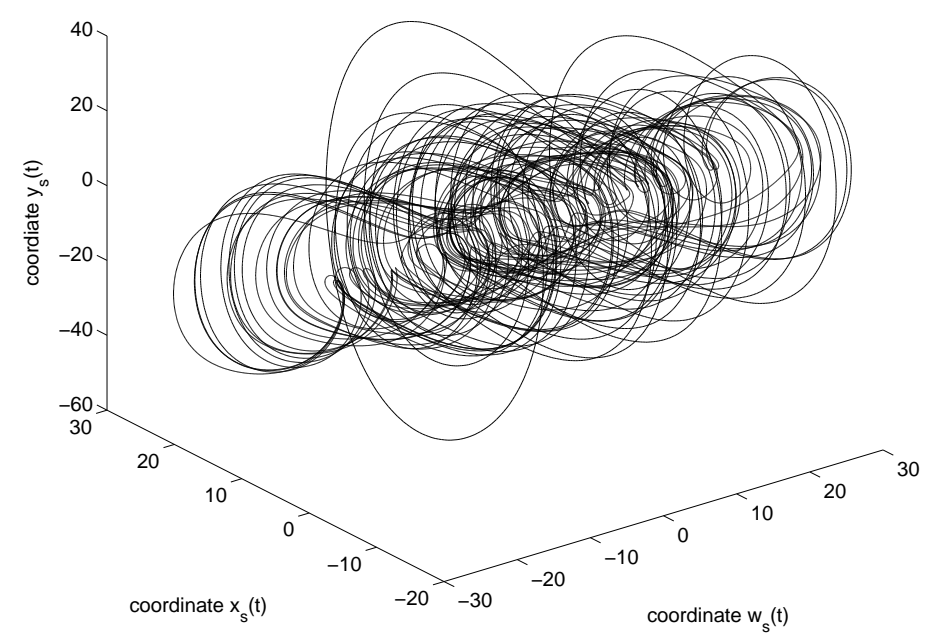

Fig. 3. Attractor of hyper-chaotic system without inputs i.e., $u_{x}=u_{y}=u_{w}=u_{z}=0$ - coordinates $w$ - $x-y$

to estimate numerically $\dot{y}$ or to use its definition from Eq. (3b). The latter imposes to know the states $x_{s}, w_{s}$ and $z_{s}$ and uses bilinear terms. In this article we show that it is possible to suppress the hyper-chaotic behaviour by acting on one single equation, that is, we only use the control input $u_{y}$. We achieve this by linear proportional feedback of the variable $x_{s}$. As a corollary of our main result we can draw the conclusion that the system may be stabilized from a chaotic regime by simply varying the parameter $b$.

\section{Control of hyper-chaotic system to the origin}

\subsection{Proportional feedback control}

We solve the problem of stabilizing the system (3) at the origin i.e., the equilibrium $\left(x_{s}, y_{s}, z_{s}, w_{s}\right)=(0,0,0,0)$. Firstly, we stress, as in [5] that the origin is a saddle point of the unforced system; this can be viewed by investigating the roots of the characteristic polynomial of the linearization of (3) around the origin:

$$
\left[\begin{array}{l}
\dot{x}_{s} \\
\dot{y}_{s} \\
\dot{z}_{s} \\
\dot{w}_{s}
\end{array}\right]=\underbrace{\left[\begin{array}{cccc}
-a & a & 0 & 0 \\
b & 0 & 0 & 1 \\
0 & 0 & -c & 0 \\
-d & 0 & 0 & 0
\end{array}\right]}_{A}\left[\begin{array}{l}
x_{s} \\
y_{s} \\
z_{s} \\
w_{s}
\end{array}\right] .
$$

The roots of the characteristic polynomial of $A$ that is, the roots of $\operatorname{det}(A-\lambda I)=0$ are

$$
\lambda_{1}=-25.7153, \quad \lambda_{2}=15.4484, \quad \lambda_{3}=-2.5, \quad \lambda_{4}=0.2668 .
$$


The following theorem establishes that the simple and unique control law $u_{y}=-k_{1} x_{s}$ in the second equation suffices to stabilize the system at the origin.

Theorem 1 Consider the system (3) and assume that all the parameters are such that the system in open loop is chaotic. Consider the controller

$$
u_{x}=0, \quad u_{y}=-k_{1} x \quad u_{z}=0, \quad u_{w}=0 .
$$

Then, for sufficiently large $k_{1}$ the zero equilibrium $(x, y, z, w)=(0,0,0,0)$ of the closedloop system is globally asymptotically stable.

Proof. We split the proof in two parts. First, we prove that the trajectories of the closedloop system are uniformly globally bounded. Second, we show global asymptotic stability. The first part of the proof relies on the observation that the system's trajectories cannot explode in finite time and, that under a chaotic (open-loop) regime, the trajectories are uniformly bounded. The second part relies on standard Lyapunov theory.

Let $\xi:=\left[x_{s}, y_{s}, z_{s}, w_{s}\right]^{\top}$ denote the state of the closed-loop system (3), (8). Correspondingly, let $\xi\left(t, \xi_{\circ}\right)$ denote the closed-loop trajectories with initial condition $\xi(0)=\xi_{\circ} \in \mathbb{R}^{4}$. We also use the shorthand notation $\xi(t)$.

Part Ia. First, we show that the system is uniformly forward complete i.e., that the solutions may not grow to infinity faster than exponentially with uniform increasing rate (no finite-time explosion is possible). Consider the functions

$$
W_{1}(\xi):=\frac{1}{2} x_{s}^{2} \quad W_{2}(\xi):=\frac{1}{2} y_{s}^{2} \quad W_{3}(\xi):=\frac{1}{2} z_{s}^{2} \quad W_{4}(\xi):=\frac{1}{2} w_{s}^{2} .
$$

Define $\omega_{i}(t):=W_{i}(\xi(t)), i \in\{1, \ldots, 4\}$ and $\omega(t):=\sum_{i=1}^{4} \omega_{i}(t)$. A simple computation shows that there exist positive numbers $\nu_{1}$ and $\nu_{2}$ (which depend on the system's parameters) such that the time derivative of $\omega(t)$ along the closed-loop trajectories satisfies

$$
\dot{\omega}(t) \leq \nu_{1} \sum_{i=1}^{4} \omega_{i}(t)+\nu_{2}\left(\omega_{1}(t)^{2}+\omega_{2}(t)^{2}+\omega_{3}(t)^{2}\right)
$$

To see this, note that (in particular) the total derivative of $W_{2}$, using (3b) and (8), yields $\dot{W}_{2}=\left(b-k_{1}\right) y_{s} x_{s}-k y_{s} x_{s} z_{s}+y_{s} w_{s}$. Hence, using the triangle inequality $a b \leq 0.5\left(a^{2}+b^{2}\right)$ on each term we see that

$$
\begin{aligned}
\dot{\omega}_{2}(t) & \leq 0.5\left(b-k_{1}\right)\left[y_{s}(t)^{2}+x_{s}(t)^{2}\right]+0.5 k\left(y_{s}(t)^{2}+\left[x_{s}(t) z_{s}(t)\right]^{2}\right)+0.5\left[y_{s}^{2}+w_{s}^{2}\right] \\
& \leq\left(b-k_{1}\right)\left[\omega_{2}(t)+\omega_{1}(t)\right]+k\left(\omega_{2}(t)+\omega_{1}(t)^{2}+\omega_{3}(t)^{2}\right)+\omega_{2}(t)+\omega_{4}(t) \\
& \leq \nu_{1}^{\prime}\left(\omega_{1}(t)+\omega_{2}(t)+\omega_{4}(t)\right)+\nu_{2}^{\prime}\left(\omega_{1}(t)^{2}+\omega_{3}(t)^{2}\right), \quad \nu_{1}^{\prime}, \nu_{2}^{\prime}>0 .
\end{aligned}
$$

Mutatis mutandis, one finds similar bounds for $\dot{\omega}_{1}, \dot{\omega}_{3}$ and $\dot{\omega}_{4}$.

Now, let $t_{\max }>0$ be such that $|\xi(t)| \rightarrow \infty$ as $t \rightarrow t_{\max }$. We proceed to evaluate the integral on both sides of the inequality in (9) from any $t^{\prime} \in\left[t_{0}, t_{\max }\right)$ to $t_{\max }$ where $t^{\prime}$ is 
such that $\omega_{i}^{\prime}:=\omega_{i}\left(t^{\prime}\right)>0$ for all $i \in\{1,2,3,4\}$. To that end we rewrite (9) as

$$
\frac{d\left(\omega_{1}+\omega_{2}+\omega_{3}+\omega_{4}\right)}{\nu_{1} \sum_{i=1}^{4} \omega_{i}+\nu_{2}\left(\omega_{1}^{2}+\omega_{2}^{2}+\omega_{3}^{2}\right)} \leq d t
$$

and define $\omega^{\max }:=\lim \sup _{t \rightarrow t_{\max }} \omega(t)$ to see that

$$
\int_{\omega^{\prime}}^{\omega^{\max }} \frac{d \omega_{4}}{\nu_{1} \sum_{i=1}^{4} \omega_{i}+\nu_{2}\left(\omega_{1}^{2}+\omega_{2}^{2}+\omega_{3}^{2}\right)} \leq \int_{t^{\prime}}^{t_{\max }} d t
$$

If any of the elements of $\xi(t):=\left[x_{s}(t), y_{s}(t), z_{s}(t), w_{s}(t)\right]^{\top}$ goes to infinity we have $\omega^{\max }=$ $+\infty$ hence, we have

$$
\left.\ln \left(\nu_{1} \omega_{4}+\nu_{1} \sum_{i=1}^{3} \omega_{i}+\nu_{2}\left(\omega_{1}^{2}+\omega_{2}^{2}+\omega_{3}^{2}\right)\right)\right|_{\omega_{4}=\omega^{\prime}} ^{\infty}=t_{\max }-t^{\prime}
$$

which implies that $t_{\max }=+\infty$. In view of the definition of $W_{4}$ we see that the trajectories $w_{s}(t)$ may not grow faster than exponentially and with a rate independent of the initial conditions. A simple inspection at the closed-loop equations (3)-(8) shows that the same holds for the other variables. We conclude that the system is uniformly forward complete.

Part $I b$. We now show boundedness. From forward completeness it follows that for each $\delta>0$ and $T \geq 0$ there exists $M(\delta, T)$ such that

$$
\left|\xi_{\circ}\right| \leq \delta \Longrightarrow|\xi(t)| \leq M(\delta, T) \quad \forall t \in[0, T] .
$$

$M$ is, in general, a non-decreasing function of its arguments. Let the system operate in open loop for all $t \in[0, T]$ for any $T>0$. Let $r$ be such that the closed ball $\bar{B}_{r}$ is the smallest compact set strictly containing the system's attractor and let $T^{*}>0$ be the smallest number such that $\xi(t) \in B_{r} \quad \forall t \geq T^{*} . T^{*}$ is finite since the system is chaotic and is independent of initial conditions since the system (3)-(8) is autonomous. We obtain that $|\xi(t)| \leq \max \{M(\delta, T), r\}$ for all $t \in[0, T]$ and any $T>0$. Define $\bar{M}:=M\left(\delta, T^{*}\right)$ then, $|\xi(t)| \leq\{\bar{M}(\delta), r\}$ for all $t \geq 0$. Note that the same holds as $T \rightarrow 0$. That is, the solutions of (3)-(8) are uniformly globally bounded.

Part II. The proof of stability relies on regarding the closed-loop system as the cascade interconnection of two systems: the first being given by (3a), (3b), (3d) and (8) with "input" $z_{s}=z_{s}(t)$ and the second being given by the $z_{s}$ equation, (3c), (8), regarded as a system with input $x_{s}(t)$. The proof is constructed in two parts. First, we show exponential stability of (3a), (3b), (3d) uniformly in bounded inputs $z_{s}(t)$. Then, we show exponential stability of $(3 \mathrm{c})$ considering that it consists of a stable filter with a decaying input.

Following up Part I define, for any $\delta>0, \beta_{x}:=\max \{\bar{M}(\delta), r\}$. Then, for any $\xi_{\circ} \in B_{\delta}$ and all $t \geq 0$,

$$
\sup _{t \geq 0}\left|x_{s}(t)\right| \leq \beta_{x}
$$


Integrating on both sides of Eq. (3c) we have

$$
z_{s}(t)=z_{s}(0) \mathrm{e}^{-c t}+\int_{0}^{t} h x_{s}(\tau)^{2} \mathrm{e}^{-c(t-\tau)} d \tau
$$

so using (10) we obtain

$$
\begin{aligned}
\left|z_{s}(t)\right| & \leq\left|z_{s}(0)\right| \mathrm{e}^{-c t}+h \beta_{x}^{2} \int_{0}^{t} \mathrm{e}^{-c(t-\tau)} d \tau \\
& \leq\left|z_{s}(0)\right|+\frac{h \beta_{x}^{2}}{c} \leq \delta+\frac{h \beta_{x}^{2}}{c}:=\beta_{z}(\delta) \quad \forall t \geq 0 .
\end{aligned}
$$

Let $\zeta^{\top}:=\left[x_{s}, y_{s}, w_{s}\right]$. Consider the functions

$$
\begin{array}{cl}
V_{1}(\zeta):=\frac{1}{2}\left(\alpha_{1} x_{s}^{2}+\alpha_{3} y_{s}^{2}+\alpha_{2} w_{s}^{2}\right), & \alpha_{1}, \alpha_{2}, \alpha_{3}>0 \\
V_{2}(\zeta):=-\varepsilon_{1} x_{s} y_{s}-\varepsilon_{2} w_{s} y_{s}, & \varepsilon_{1}, \quad \varepsilon_{2}>0 .
\end{array}
$$

The function $V(\zeta):=V_{1}(\zeta)+V_{2}(\zeta)$ is positive definite, radially unbounded and satisfies

$$
\left(\left[\begin{array}{l}
x_{s} \\
y_{s}
\end{array}\right]^{\top} A_{1}\left[\begin{array}{l}
x_{s} \\
y_{s}
\end{array}\right]+\left[\begin{array}{l}
w_{s} \\
y_{s}
\end{array}\right]^{\top} A_{2}\left[\begin{array}{l}
w_{s} \\
y_{s}
\end{array}\right]\right) \geq V(\zeta) \geq \frac{1}{2}\left(\left[\begin{array}{l}
x_{s} \\
y_{s}
\end{array}\right]^{\top} A_{1}\left[\begin{array}{l}
x_{s} \\
y_{s}
\end{array}\right]+\left[\begin{array}{l}
w_{s} \\
y_{s}
\end{array}\right]^{\top} A_{2}\left[\begin{array}{l}
w_{s} \\
y_{s}
\end{array}\right]\right)
$$

where the eigen-values of the matrices

$$
A_{1}:=\left[\begin{array}{cc}
\alpha_{1} & -\varepsilon_{1} \\
-\varepsilon_{1} & \frac{\alpha_{3}}{2}
\end{array}\right], \quad A_{2}:=\left[\begin{array}{cc}
\alpha_{2} & -\varepsilon_{2} \\
-\varepsilon_{2} & \frac{\alpha_{3}}{2}
\end{array}\right]
$$

are bounded from above and below by positive numbers for appropriate values of $\alpha_{1}$ and $\alpha_{2}$. The total time derivative of $V_{1}$ along the closed-loop trajectories yields

$$
\dot{V}_{1}(\zeta)=-\alpha_{1} a x_{s}^{2}+\alpha_{1} a y_{s} x_{s}+\alpha_{3} b x_{s} y_{s}-\alpha_{3} k x_{s} y_{s} z_{s}+\alpha_{3} w_{s} y_{s}-\alpha_{3} k_{1} x_{s} y_{s}-\alpha_{2} d w_{s} x_{s} .
$$

Let

$$
\alpha_{1}:=\frac{\alpha_{3}\left(k_{1}-b\right)}{a}
$$

then, for all $\left|z_{s}\right| \leq \beta_{z}$,

$$
\begin{aligned}
\dot{V}_{1}(\zeta) & =-\alpha_{1} a x_{s}^{2}-\alpha_{3} k x_{s} y_{s} z_{s}+\alpha_{3} w_{s} y_{s}-\alpha_{2} d w_{s} x_{s} \\
& \leq-\alpha_{1} a x_{s}^{2}+\alpha_{3} k \beta_{z}\left|x_{s}\right|\left|y_{s}\right|+\alpha_{3} w_{s} y_{s}-\alpha_{2} d w_{s} x_{s}
\end{aligned}
$$


The total time derivative of $V_{2}$ along the closed-loop trajectories yields

$$
\begin{aligned}
\dot{V}_{2}(\zeta)= & -\left[b x_{s}-k x_{s} z_{s}+w_{s}-k_{1} x_{s}\right]\left[\varepsilon_{1} x_{s}+\varepsilon_{2} w_{s}\right]-y_{s}\left[\varepsilon_{1} a\left(y_{s}-x_{s}\right)-\varepsilon_{2} d x_{s}\right] \\
\leq & -\varepsilon_{1}\left[a\left(y_{s}-x_{s}\right)\right] y_{s}-\left[\varepsilon_{1} x_{s}+\varepsilon_{2} w_{s}\right]\left[\left(b-k_{1}\right) x_{s}-k x_{s} z_{s}+w_{s}\right]+\varepsilon_{2} d x_{s} y_{s} \\
\leq & -\varepsilon_{1}\left(b-k_{1}\right) x_{s}^{2}-\varepsilon_{2}\left(b-k_{1}\right) w_{s} x_{s}+\varepsilon_{1} k \beta_{z} x_{s}^{2}-\varepsilon_{1} w_{s} x_{s}+\varepsilon_{2} k \beta_{z}\left|x_{s}\right|\left|w_{s}\right| \\
& \quad-\varepsilon_{2} w_{s}^{2}-\varepsilon_{1} a y_{s}^{2}+\varepsilon_{1} a x_{s} y_{s}+\varepsilon_{2} d x_{s} y_{s} .
\end{aligned}
$$

Putting together (14) and (15) we obtain

$$
\begin{aligned}
\dot{V}_{1}+\dot{V}_{2} \leq- & x_{s}^{2}\left[\alpha_{1} a-\varepsilon_{1}\left(k \beta_{z}+k_{1}-b\right)\right]-\varepsilon_{2} w_{s}^{2}-\varepsilon_{1} a y_{s}^{2}+w_{s} x_{s}\left[\varepsilon_{2}\left(k_{1}-b\right)-\varepsilon_{1}-\alpha_{2} d\right] \\
& +\varepsilon_{2} k \beta_{z}\left|x_{s}\right|\left|w_{s}\right|+\left(\varepsilon_{1} a+\varepsilon_{2} d\right) x_{s} y_{s}+\alpha_{3} k \beta_{z}\left|x_{s}\right|\left|y_{s}\right|+\alpha_{3} w_{s} y_{s}
\end{aligned}
$$

For any $d>0$ and $k_{1}>b$ we define

$$
\alpha_{2}:=\frac{\varepsilon_{2}\left(k_{1}-b\right)-\varepsilon_{1}}{d}
$$

and we impose

$$
\frac{\alpha_{1} a}{2} \geq \varepsilon_{1}\left(k \beta_{z}+k_{1}-b\right)
$$

which, in view of (13) holds if and only if

$$
\varepsilon_{1} \leq \frac{\alpha_{3}\left(k_{1}-b\right)}{k \beta_{z}+k_{1}-b}
$$

which holds for $\varepsilon_{1}$ sufficiently small. Under these conditions we obtain

$$
\begin{aligned}
\dot{V}_{1}+\dot{V}_{2} \leq- & \frac{\alpha_{1} a}{2} x_{s}^{2}-\varepsilon_{2} w_{s}^{2}-\varepsilon_{1} a y_{s}^{2} \\
& +\varepsilon_{2} k \beta_{z}\left|x_{s}\right|\left|w_{s}\right|+\left(\varepsilon_{1} a+\varepsilon_{2} d+\alpha_{3} k \beta_{z}\right)\left|x_{s}\right|\left|y_{s}\right|+\alpha_{3} w_{s} y_{s}
\end{aligned}
$$

which holds if

$$
\begin{aligned}
& \dot{V}_{1}+\dot{V}_{2} \leq-\frac{1}{3} \varepsilon_{1} a y_{s}^{2}-\frac{1}{6} \alpha_{1} a x_{s}^{2}-\frac{1}{3} \varepsilon_{2} w_{s}^{2} \\
& -\left[\begin{array}{l}
\left|x_{s}\right| \\
\left|w_{s}\right|
\end{array}\right]^{\top}\left[\begin{array}{cc}
\frac{\alpha_{1} a}{6} & -\frac{\varepsilon_{2} k \beta_{z}}{2} \\
-\frac{\varepsilon_{2} k \beta_{z}}{2} & \frac{\varepsilon_{2}}{3}
\end{array}\right]\left[\begin{array}{l}
\left|x_{s}\right| \\
\left|w_{s}\right|
\end{array}\right]-\left[\begin{array}{c}
\left|w_{s}\right| \\
\left|y_{s}\right|
\end{array}\right]^{\top}\left[\begin{array}{cc}
\frac{\varepsilon_{2}}{3} & -\frac{\alpha_{3}}{2} \\
-\frac{\alpha_{3}}{2} & \frac{\varepsilon_{1} a}{3}
\end{array}\right]\left[\begin{array}{l}
\left|w_{s}\right| \\
\left|y_{s}\right|
\end{array}\right] \\
& -\left[\begin{array}{l}
\left|x_{s}\right| \\
\left|y_{s}\right|
\end{array}\right]^{\top}\left[\begin{array}{cc}
\frac{\alpha_{1} a}{6} & -\frac{1}{2}\left(\varepsilon_{1} a+\varepsilon_{2} d+\alpha_{3} k \beta_{z}\right) \\
-\frac{1}{2}\left(\varepsilon_{1} a+\varepsilon_{2} d+\alpha_{3} k \beta_{z}\right) & \frac{\varepsilon_{1} a}{3}
\end{array}\right]\left[\begin{array}{l}
\left|x_{s}\right| \\
\left|y_{s}\right|
\end{array}\right]
\end{aligned}
$$

The matrices above are symmetric and positive semidefinite under the following conditions, respectively:

cond 1. $\quad \frac{\alpha_{1} a}{9} \geq \frac{\varepsilon_{2} k^{2} \beta_{z}^{2}}{2}$ 
cond 2. $\quad \frac{\varepsilon_{1} \varepsilon_{2} a}{9} \geq \frac{\alpha_{3}^{2}}{4}$

cond 3. $\quad \frac{\varepsilon_{1} \alpha_{1} a^{2}}{9} \geq \frac{1}{2}\left(\varepsilon_{1} a+\varepsilon_{2} d+\alpha_{3} k \beta_{z}\right)^{2}$

For any fixed values of the other constants involved, conditions 1 and 3 hold for sufficiently large $\alpha_{1}$ which, in view of (13), is possible by enlarging the control gain $k_{1}$. On the other hand, note that both the denominator and the numerator of (19) are of linear order in $k_{1}$ hence, enlarging the latter does not violate (19) which is fulfilled for $\varepsilon_{1}$ sufficiently small. Also, choices of large $k_{1}$ and small $\varepsilon_{1}$ do not violate the positivity of $\alpha_{2}-c f$. Eq. (17). Furthermore, for any (small) $\varepsilon_{1}$ Condition 2 is met if $\alpha_{3}$ is small enough i.e., if

$$
\alpha_{3} \leq \frac{2}{3} \sqrt{\varepsilon_{1} \varepsilon_{2} a}
$$

which, in view of (19) imposes that

$$
\varepsilon_{1} \leq \frac{4 \varepsilon_{2} a\left(k_{1}-b\right)^{2}}{9\left[k \beta_{z}+k_{1}-b\right]^{2}} .
$$

The latter holds for small values of $\varepsilon_{1}$ and an appropriate choice of $\varepsilon_{2}$.

In summary, for sufficiently large $k_{1}$, there exist positive reals $\varepsilon_{1}, \varepsilon_{2}, \alpha_{1}, \alpha_{3}$ such that $\dot{V}(\zeta):=\dot{V}_{1}(\zeta)+\dot{V}_{2}(\zeta)$ is negative definite and satisfies

$$
\dot{V}(\zeta) \leq-\frac{1}{3} \varepsilon_{1} a y_{s}^{2}-\frac{1}{6} \alpha_{1} a x_{s}^{2}-\frac{1}{3} \varepsilon_{2} w_{s}^{2}
$$

where $\varepsilon_{1}, \varepsilon_{2}$ and $\alpha_{1}$ depend (implicitly or explicitly) of $\beta_{z}$. The latter holds for each fixed $\beta_{z}(\delta)$ hence, for each $\delta>0$. From standard Lyapunov theory i.e., integrating on both sides of (22) from 0 to $t$ and using (12) - positive definiteness of $V(\zeta)$ - we obtain the existence of $\kappa$ and $\lambda>0$ depending on $k_{1}$ and $\delta$, such that

$$
|\zeta(t)| \leq \kappa|\zeta(0)| \mathrm{e}^{-\lambda t} \quad \forall t \geq 0, \delta>0,|\xi(0)| \leq \delta
$$

Using this and the fact that $x_{s}(t)^{2} \leq|\zeta(t)|^{2}$ we obtain from (11):

$$
\begin{aligned}
\left|z_{s}(t)\right| & \leq\left|z_{s}(0)\right| \mathrm{e}^{-c t}+\int_{0}^{t} h|\xi(0)|^{2} \mathrm{e}^{-2 \lambda \tau} \mathrm{e}^{-c(t-\tau)} d \tau \\
& \leq\left|z_{s}(0)\right| \mathrm{e}^{-c t}+\int_{0}^{t} h|\xi(0)|^{2} \mathrm{e}^{(c-2 \lambda) \tau} \mathrm{e}^{-c t} d \tau \\
& \leq\left|z_{s}(0)\right| \mathrm{e}^{-c t}+\frac{1}{c-2 \lambda} h|\xi(0)|^{2} \mathrm{e}^{-c t} \int_{0}^{t} \mathrm{e}^{(c-2 \lambda) \tau}(c-2 \lambda) d \tau \\
& \leq\left|z_{s}(0)\right| \mathrm{e}^{-c t}+\frac{1}{c-2 \lambda} h|\xi(0)|^{2} \mathrm{e}^{-c t} \mathrm{e}^{(c-2 \lambda) t} \\
& \leq\left|z_{s}(0)\right| \mathrm{e}^{-c t}+\frac{1}{c-2 \lambda} h|\xi(0)|^{2} \mathrm{e}^{-2 \lambda t}
\end{aligned}
$$

Since the latter holds for each $\delta>0$ and all $|\xi(0)| \leq \delta$ we conclude global asymptotic attractivity of the zero equilibrium $\left(x_{s}, y_{s}, z_{s}, w_{s}\right)=(0,0,0,0)$. Furthermore, from the 
previous computations $-c f$. Ineq. (22), it readily follows that the origin $\xi=0$ is Lyapunov (locally) stable. This, and that the closed-loop system's trajectories are globally bounded imply the theorem's statement.

\subsubsection{Simulation experiments}

We have tested the controller in simulations using parameters from the literature. The system parameters are set as in (4) and the initial conditions are set as in (5) so the hyper-chaotic 4th order system is in chaotic regime. The system is left to evolve freely under chaotic regime for 50s. Then, the control action starts at this moment. The numeric simulations results are showed in Figures $4-7$. One can clearly appreciate the exponential convergence and fast transient after $T=50 \mathrm{~s}$.
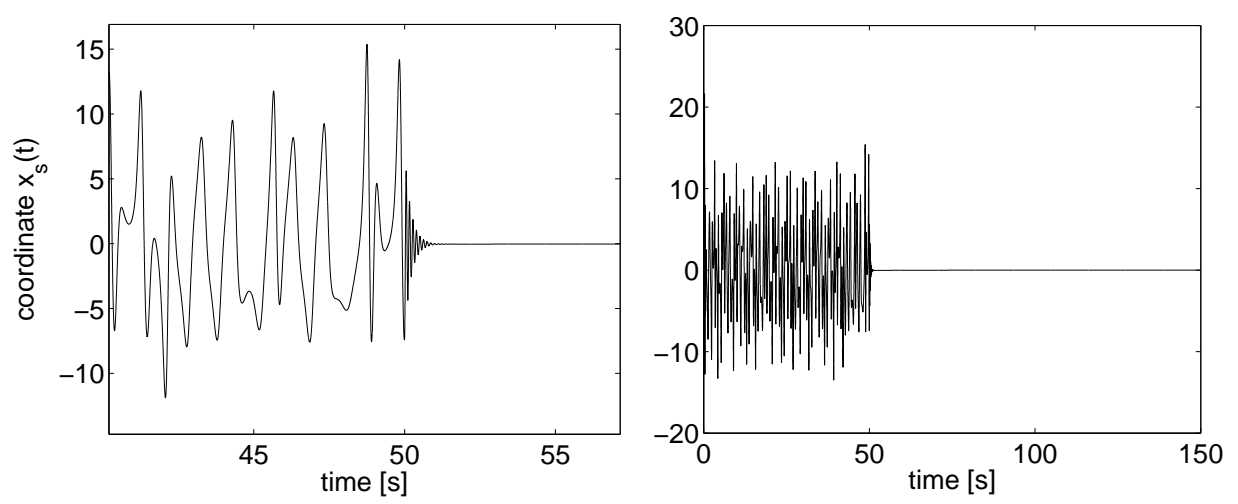

Fig. 4. Integral curve of coordinate $x_{s}(t)$, control action starts at $t=50 \mathrm{~s}$
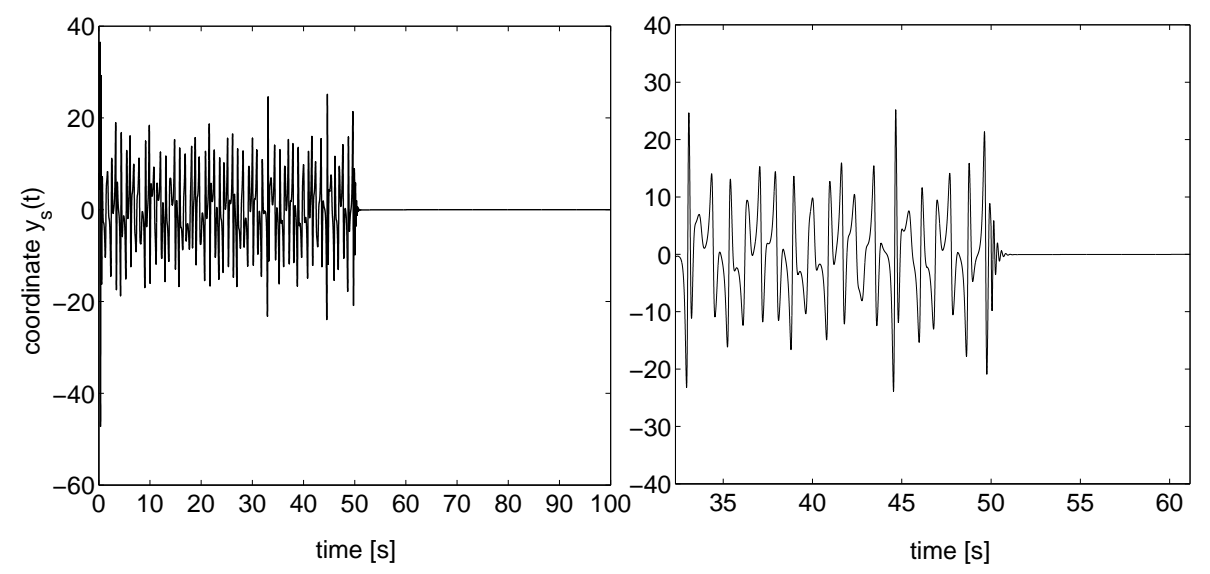

Fig. 5. Integral curve of coordinate $y_{s}(t)$, control action starts at $t=50 \mathrm{~s}$

\subsection{Stabilization via parameter variation}

As is clear from Theorem 1 and the simulation experiments, suppression of hyper-chaotic behaviour hinges upon the ability of introducing the term $-k_{1} x_{s}$ in the second equation. For sufficiently large values of $k_{1}$, notably $k_{1}>b$ this control term dominates over the 

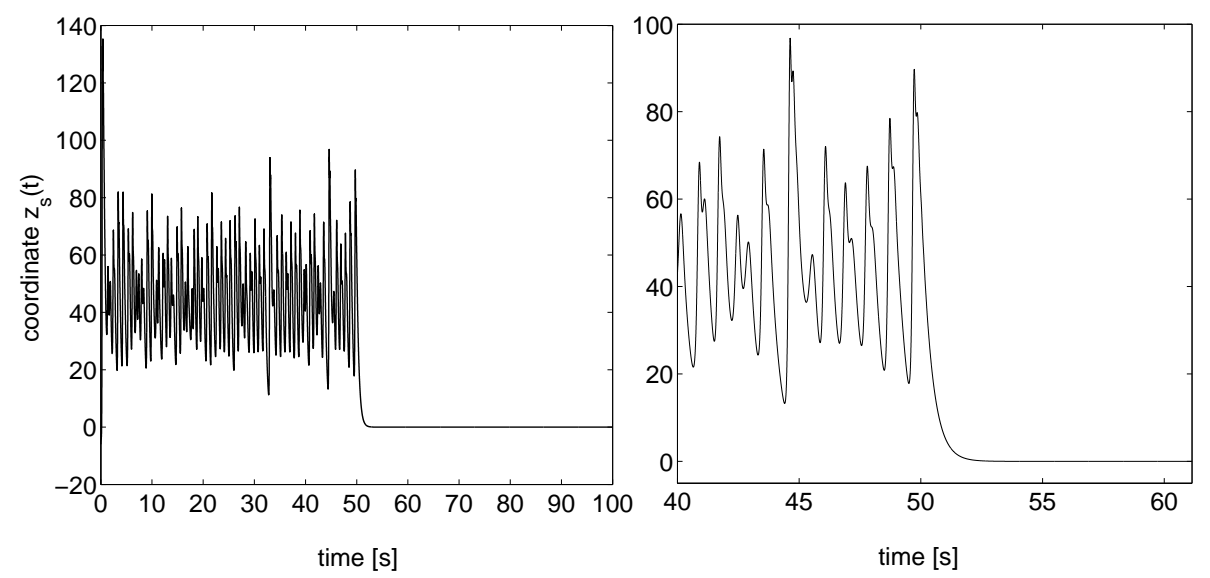

Fig. 6. Integral curve of coordinate $z_{s}(t)$, control action starts at $t=50 \mathrm{~s}$
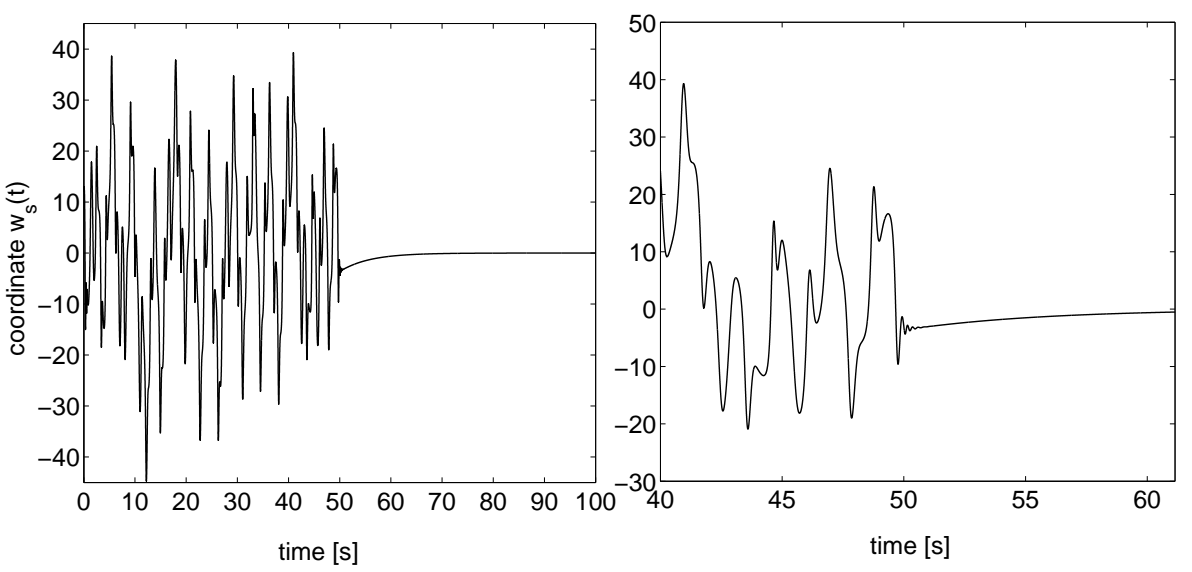

Fig. 7. Integral curve of coordinate $w_{s}(t)$, control action starts at $t=50 \mathrm{~s}$

term $b x_{s}$ in $(3 \mathrm{~b})$. To better understand the stabilization mechanism at an intuitive level, let us rewrite the closed-loop system in the following form:

$$
\begin{aligned}
{\left[\begin{array}{c}
\dot{x}_{s} \\
\dot{y}_{s} \\
\dot{w}_{s}
\end{array}\right] } & =\left[\begin{array}{lll}
-a & a & 0 \\
\gamma(t) & 0 & 1 \\
-d & 0 & 0
\end{array}\right]\left[\begin{array}{l}
x_{s} \\
y_{s} \\
w_{s}
\end{array}\right] \\
\dot{z}_{s} & =-c z_{s}+h x_{s}^{2} \\
\gamma(t) & =b-k_{1}-k z_{s}(t)
\end{aligned}
$$

Since $z_{s}(t)$ is bounded while under chaotic regime, the system of equations (25a) may be viewed as a linear time-varying system with time varying parameter $\gamma(t)$. Note that if $\gamma$ were constant, it is easy to impose conditions on this value so that the eigen-values of the matrix in (25a) are negative and therefore the system (25a) is exponentially stable at the origin. 
As for Equation 25b we may interpret it as the equation of a low-pass filter, an exponentially stable system with bounded input $x_{s}(t)$. Moreover, if $x_{s}(t)$ tends to zero (exponentially), so does $z_{s}(t)$ (exponentially). Since $z_{s}(t)$ is bounded in chaotic regime, it is clear that one may vary the parameter $\gamma(t)$ by varying the parameter $k_{1}$ to ensure that $\gamma(t)$ is sufficiently negative after sufficiently long time. If $\gamma$ satisfies such characteristic it is possible to show the exponential stability of the system (25) as well it is possible to induce chaotic behaviour by simply tuning this same parameter. The proof of this statement follows closely the proof of Theorem 1 and shall not be repeated.
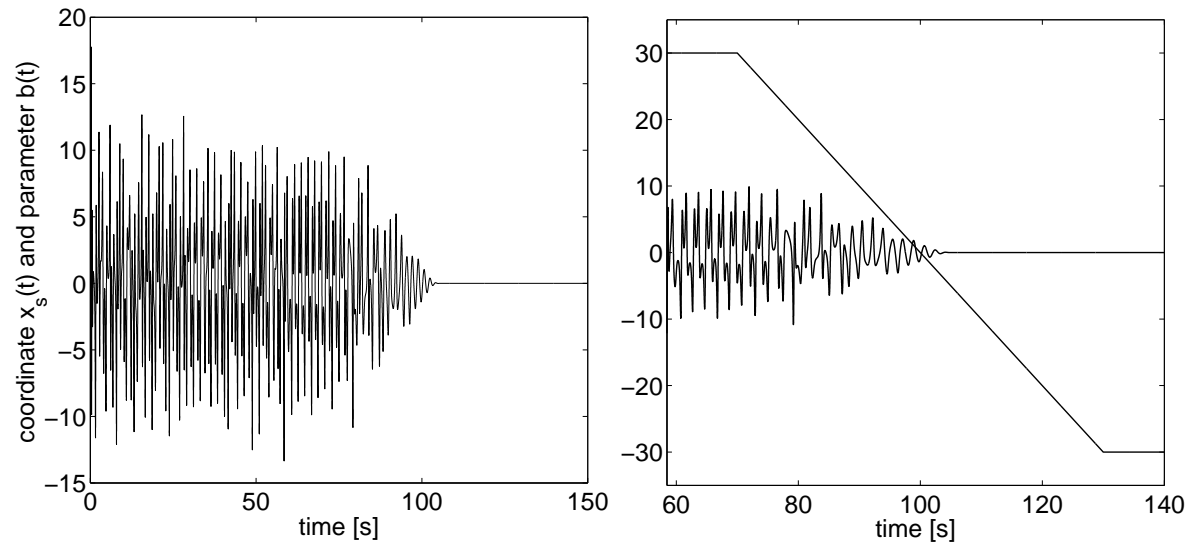

Fig. 8. Integral curve of coordinate $x_{s}(t)$ with time-varying parameter $b(t)$ which evolves linearly from +40 to -20 between $\mathrm{t}=70 \mathrm{~s}$ and $\mathrm{t}=130 \mathrm{~s}$.
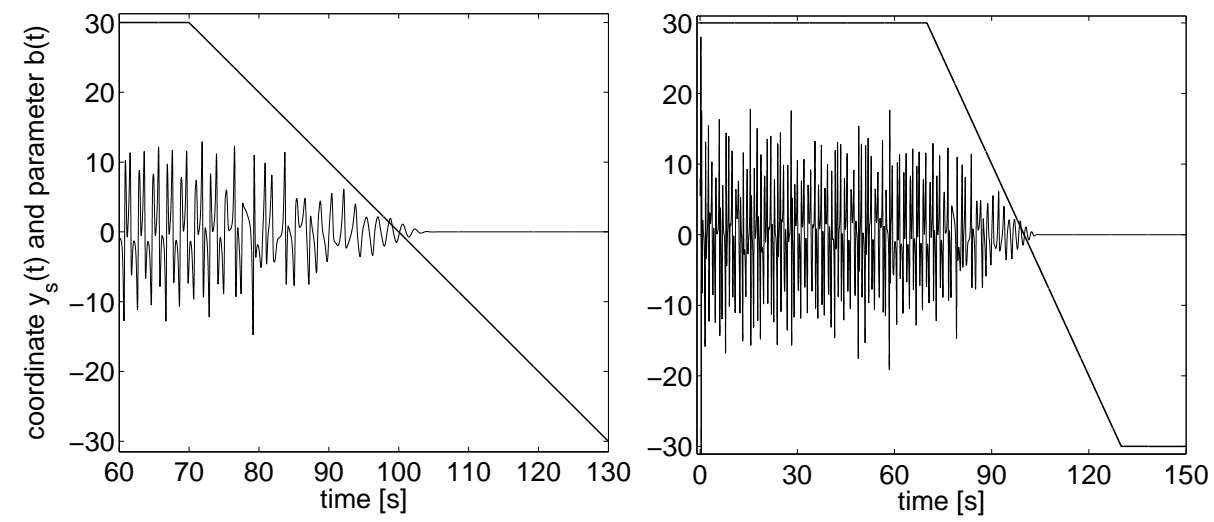

Fig. 9. Integral curve of coordinate $y_{s}(t)$ with time-varying parameter $b(t)$ which evolves linearly from +40 to -20 between $\mathrm{t}=70 \mathrm{~s}$ and $\mathrm{t}=130 \mathrm{~s}$.

For illustration we have performed more numeric simulations. For that purpose, redefine $b(t):=b-k_{1}(t)$ where $b$ is the original nominal constant parameter of system (2) (in this simulation $b=40)$ and $k_{1}$ is a part that is left to vary from 0 to +60 so $b(t)$ decays linearly from +40 to -20 over $60 \mathrm{~s}$. The response of the system with such $b(t)$ is showed in Figures 8-11. One can see that all the system's coordinates exponentially converge to zero as $b(t)$ becomes sufficiently negative. As is shown in the figure, the final value of $b(t)$ is $b=-20$. That is, modifying the parameter $b$ to appropriate values tantamounts to applying the feedback control from Theorem 1. 

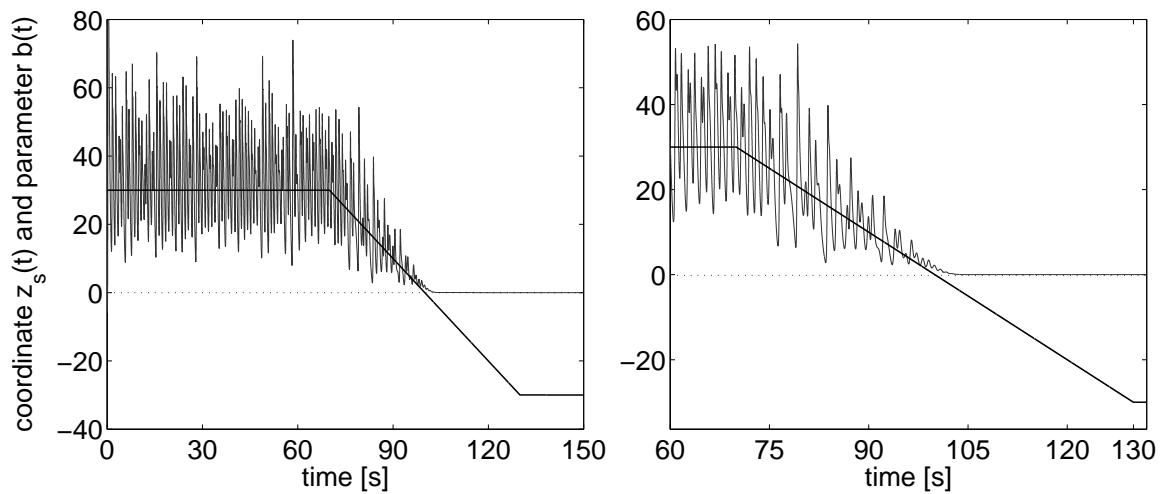

Fig. 10. Integral curve of coordinate $z_{s}(t)$ with time-varying parameter $b(t)$ which evolves linearly from +40 to -20 between $\mathrm{t}=70 \mathrm{~s}$ and $\mathrm{t}=130 \mathrm{~s}$.
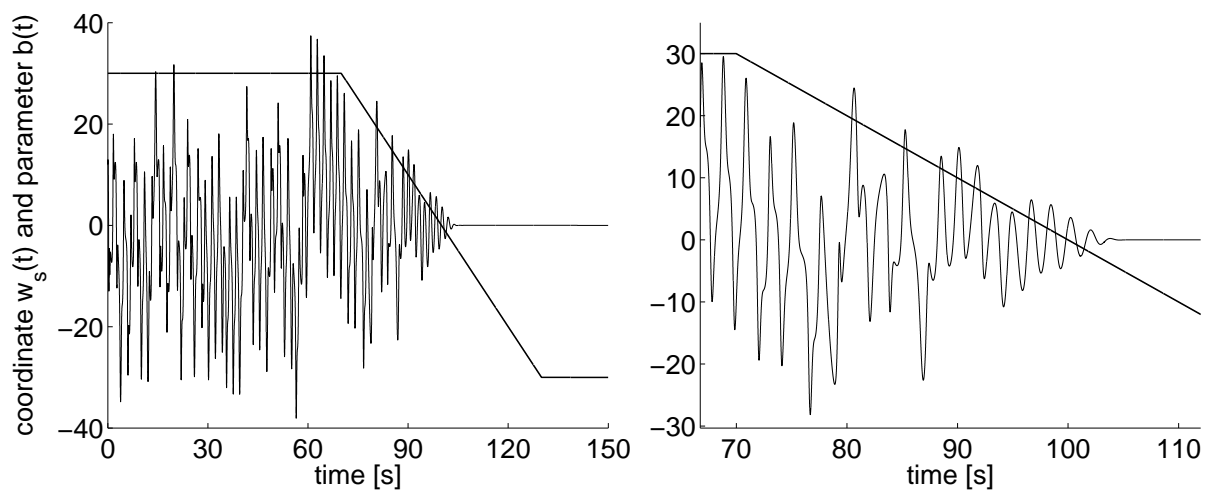

Fig. 11. Integral curve of coordinate $w_{s}(t)$ with time-varying parameter $b(t)$ which evolves linearly from +40 to -20 between $\mathrm{t}=70 \mathrm{~s}$ and $\mathrm{t}=130 \mathrm{~s}$.

\section{Conclusions}

We have showed that suppression of chaos, in particular, control to the zero equilibrium is possible for the hyper-chaotic system of Lü via one control input. Our control law is in it's simplest possible form; proportional feedback. This can also be viewed as control via varying one of the system parameters.

\section{References}

[1] M. Ayati and A. Khaki-Sedigh. Adaptive control of nonlinear in parameters chaotic system via Lyapunov exponents placement. Chaos, Solitons and Fractals, 2008.

[2] J.-F. Chang, M.-L. Hung, Y.S. Yang, T.-L. Liao, and J.-J. Yan. Controlling chaos of the family of ro ssler systems using sliding mode control. Chaos, Solitons and Fractals, 37:609-622, 2008.

[3] G. Chen and t. Ueta. Yet another chaotic attractor. Int. J. of Bifurcat. and Chaos, 9:1465-1466, 1999.

[4] H. H. Chen. Chaos control and global synchronization of liu chaotic systems using linear balanced feedback control. Chaos, Solitons and Fractals, 2007. In Press, available online: 29 septembre 2007.

[5] F. Dou, J. Sun, and K. Lü W. Duan. Controlling hyperchaos in the new hyperchaotic system. Communicat Nonlinear Sci Numer Simulat, 14:552-559, 2009. 
[6] A. Y. Goharrizi, A. Khaki-Sedigh, and N. Sepehri. Observer-based adaptive control of chaos in nonlinear discrete-time systems using time-delayed state feedback. Chaos, Solitons and Fractals, 2008.

[7] D. Li and Z. Yin. Connecting the Lorenz and Chen systems via nonlinear control. Communications in Nonlinear Science and Numerical Simulation, 14:655-667, 2009.

[8] E. N. Lorenz. Deterministic nonperiodic flow. J. Atmos. Sci., 20:130-141, 1963.

[9] J. Lü, G. Chen, and D. Cheng. A new chaotic system and beyond: the generalized Lorenz-like system. Int. J. of Bifurcat. and Chaos, pages 1507-1537, 2004.

[10] J. H. Lü and G. Chen. A new chaotic attractor coined. Int. J. of Bifurcat. and Chaos, pages 659-661, 2002.

[11] A. N. Njah and O. D. Sunday. Generalization on the chaos control of 4-d chaotic systems using recursive backstepping nonlinear controller. Chaos, Solitons and Fractals, 2008.

[12] E. Ott, C. Grebogi, and J. A. Yorke. Controlling chaos. Phys. Rev. Lett., 64:1196-1199, 1990.

[13] J. H. Park. Chaos synchronization of a chaotic system via nonlinear control. Chaos, Solitons and Fractals, 25:579-584, 2005.

[14] O. E. Rössler. An equation for hyperchaos. Phys. Lett. A, 71(2-3):155-157, 2007.

[15] T. Ueta and G. Chen. Bifurcation analysis of chen's attractor. Int. J. of Bifurcat. and Chaos, 10:1917-1931, 2000.

[16] Q. Zhang and J. Lu. Chaos synchronization of a new chaotic system via nonlinear control. Chaos, Solitons and Fractals, 37:175-179, 2008. 